\title{
Chemical Kinetic Performance Losses for a Hydrogen Laser Thermal Thruster
}

\author{
T. Dwayne McCay* \\ University of Tennessee Space Institute, Tullahoma, Tennessee \\ and \\ Carol E. Dexter $\dagger$ \\ Environmental Research Institute of Michigan, Ann Arbor, Michigan
}

\begin{abstract}
Projected requirements for efficient, economical orbit-raising propulsion systems have generated investigations into several potentially high-specific-impulse, moderate-thrust advanced systems. One of these systems, laser thermal propulsion, utilizes a high-temperature plasma as the enthalpy source. The plasma is sustained by a focused laser beam that maintains the plasma temperature at levels near $15,000 \mathrm{~K}$. Since such temperature levels lead to total dissociation and high ionization, the plasma thruster system potentially has a high-specific-impulse decrement due to recombination losses. The nozzle flow is expected to be sufficiently non-equilibrium to warrant concern over the achievable specific impulse. This investigation was an attempt to evaluate those losses. The onedimensional kinetics option of the two-dimensional kinetics computer program was used with an available literature-based chemical kinetics rate set to determine the chemical kinetics energy losses for typical plasma thruster conditions. The rates were varied about the nominal accepted values to bound the possible losses. Kinetic losses were shown to be highly significant for a laser thermal thruster using hydrogen. A $30 \%$ reduction in specific impulse is possible, due simply to the inability to completely extract molecular recombination energy.
\end{abstract}

\section{Introduction}

$\mathbf{T}$ HE NASA George C. Marshall Space Flight Center (MSFC) has been involved in research of the physical phenomena associated with implementation of an advanced propulsion concept for orbit raising, i.e., laser thermal propulsion. The concept employs a single propellant, in all probability hydrogen, that is not combusted, but has its enthalpy increased by energy addition from a beamed energy source, a

Presented as Paper 85-0907 at the AIAA 20th Thermophysics Conference, Williamsburg, VA, June 19-21, 1985; revision received Nov. 1,1986 . This paper is declared a work of the U.S. Government and is not subject to copyright protection in the United States.

${ }^{*}$ Professor, Engineering Science.

$\nmid$ Research Engineer. Both authors formerly with NASA George C. Marshall Space Flight Center, Alabama. laser. The energy addition can take place through a variety of different absorption mechanisms: blackbody, molecular, or plasma. Using the plasma absorption method, a plasma is initially created by some auxiliary scheme and then sustained by the laser beam continuum absorption process, inverse Bremsstrahlung. For hydrogen using a $\mathrm{CO}_{2}(10.6 \mu \mathrm{m}$ wavelength) laser beam, the maximum plasma temperature obtained in such a process will be near $17,400 \mathrm{~K}$, the peak in the absorption coefficient/temperature curve. MSFC research has been directed toward developing a reliable plasma initiation scheme and measuring the beam absorption efficiency of hydrogen plasmas as a function of gas flow parameters. The initial results from these experiments ${ }^{1}$ indicate a high absorption efficiency and give strong support to the conjecture that a $2500 \mathrm{~s}$ specific impulse can be achieved for laser thermal rockets operating at moderate thrust levels $(5-1000 \mathrm{~N})$.

The $2500 \mathrm{~s}$ specific impulse value is based upon an efficient use of the resulting hydrogen propellant enthalpy. Unfortunately, the plasma is expected to represent only a small fraction of the total chamber volume and the losses associated with having such a small, extremely hot core flow can be large. As part of the MSFC efforts, it has been a goal to estimate these losses. Attempts at applying state-of-the-art computational models to the problem have met with minimal success. ${ }^{2}$ As a result, a set of analyses were undertaken to individually characterize the various losses; nonuniform flow, boundary layer, chemical kinetic, etc., based upon limited knowledge of the flowfield. This paper represents the results of the initial study, the effect of chemical nonequilibrium losses on the thruster performance.

For low-pressure, pure hydrogen flows, the chemical kinetic reaction system is simple. Even at high temperatures, the system of reactions is small and, therefore, the range of uncertainty is determined by a limited number of rates. Unfortunately, the uncertainties in the chemical kinetic rates for the temperature and pressure regimes of interest in laser thermal thrusters are large. For this reason, the study was conducted as a sensitivity study, where the best available rates, obtained by literature search and personal queries, were varied based upon their estimated uncertainties. These rates were applied to a single thruster design for a range of operating parameters 
estimated to be typical of a full-scale system. The premise used in the study was that a bulk equilibrium temperature could be defined and one-dimensional kinetic calculations performed which would be representative of the kinetic losses. This bulk temperature was treated as a variable to evaluate the range of losses that could be anticipated. In addition, pressure, temperature, nozzle area ratio, nozzle half-angle, and the rates themselves were varied to complete the assessment. Specific impulse was used as the central evaluation criteria.

\section{Analysis}

\section{Thruster Design/Definition}

Figure 1 is a conceptual schematic diagram of a hydrogen laser thermal thruster. The laser beam, from either a spacebased or Earth-based laser source, is brought to a focus in the absorption chamber of the thruster. Hydrogen, which is also

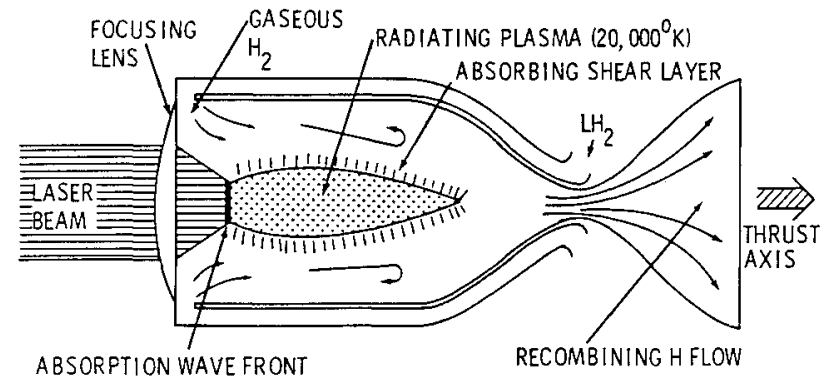

Fig. 1 Schematic diagram of a hydrogen-fueled laser thermal thruster.

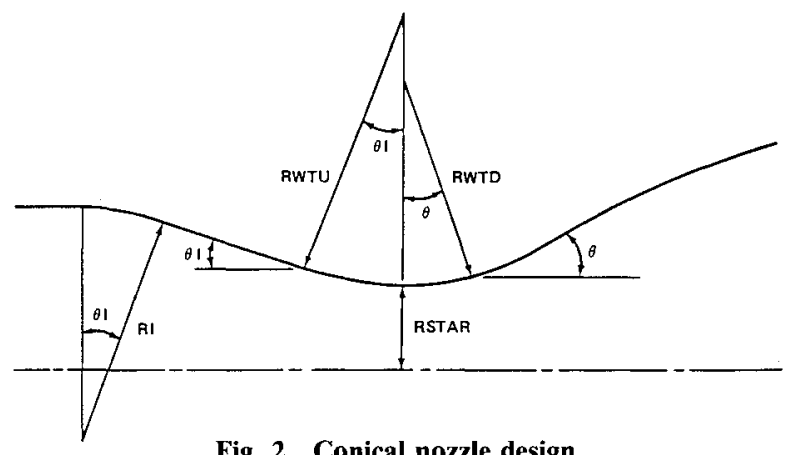

Fig. 2 Conical nozzle design.

Table 1 Conical nozzle parameters

\begin{tabular}{llll}
\hline \hline Parameters & Nozzle 1 & Nozzle 2 & Nozzle 3 \\
\hline$R S T A R, \mathrm{~cm}$ & 5.08 & 5.08 & 5.08 \\
$R W T D$ & 6.78 & 6.78 & 6.78 \\
$R W T U$ & 1.91 & 1.91 & 1.91 \\
$R I$ & 5.16 & 5.16 & 5.16 \\
$\theta I$, deg & 21 & 21 & 21 \\
$\theta$, deg & 21 & 14 & 7 \\
$C R$ & 4 & 4 & 4 \\
\hline
\end{tabular}

used to cool the thruster, enters the absorption chamber and part of it flows through the plasma region where the hydrogen absorbs energy directly from the focused beam. The plasma region is somewhat analogous to the combustion region that would exist in a conventional system. The high-temperature plasma transfers heat to the surrounding hydrogen, chamber walls, and optical window. Expansion of the high-temperature hydrogen provides the thrust. A more detailed discussion of laser thermal thruster systems and components has been given by Jones and Keefer. ${ }^{3}$

Once the combustion chamber fluid in characterized, the problem in investigating kinetic effects reverts to a simple nonequilibrium throat/nozzle flow problem. The significance of the kinetic effects were studied using three conical nozzles, highlighted in Fig. 2. Table 1 provides the design parameters for the nozzles. The cone angle and the area ratio were the variable parameters chosen for the study. The nozzle design and radii are typical for small thrusters. The throat area was chosen to provide the mass flow rate $(0.05 \mathrm{~kg} / \mathrm{s})$ required to produce choked flow at a thrust of $890 \mathrm{~N}(200 \mathrm{lbf})$ with a stagnation temperature of $5000 \mathrm{~K}$. The upper limit to the stagnation temperature may be greater than $5000 \mathrm{~K}$, but that value is consistent with practical heat loads for a small thruster utilizing $\mathrm{LH}_{2}$ transpiration cooling. Although the plasma results in an increased temperature in the center of the chamber, the one-dimensional calculation inherently assumes a flat, uniform thermal profile. The effect of two- and threedimensionality is to be included in future analyses. It is recognized that these effects will be significant and an effective mixing approach must be developed to take advantage of the thruster's true potential.

\section{Rate Equations}

A number of elementary reactions influencing the thruster performance occur in the nonequilibrium hydrogen plasma system. The importance of a given reaction within a set is a function of the chemical kinetic rate at the plasma temperature and its effect on the useful enthalpy. The effects of the kinetic rates were evaluated using the one-dimensional kinetics (ODK) option of the two-dimensional kinetics (TDK) computer code. ${ }^{4}$ An extensive literature search was conducted to determine the reactions with significant influence in the system. These reactions, with their accompanying rates, are presented in Table 2 . Several hydrogen reactions, not included in Table 2, were excluded from the analysis because they were estimated to play an insignificant role due to their very slow reaction rates and miniscule species concentrations. Atomic third-body efficiencies were generally assumed to be unity. Table 2 presents forward reaction rates for the reactions used in the analysis. The rate constant $K$ is in the units $\mathrm{cm}^{6} / \mathrm{mol}^{2} \cdot \mathrm{s}$. As previously mentioned, the chemical kinetic rates were chosen based upon the best data available from literature search and personal communications. Performance calculations were made for equilibrium, frozen, and kinetic expansions. For the kinetic expansions, two rate sets, 1 and 2, were used. Rate set 1 employed baseline (slower) or worse-case rates from a performance viewpoint. This rate set represents the currently accepted values as can be determined from available

Table 2 High-temperature hydrogen rate sets

\begin{tabular}{lll}
\hline \multicolumn{1}{c}{ Reaction } & \multicolumn{1}{c}{ Rate set 1 } & \multicolumn{1}{c}{ Rate set 2} \\
\hline $\mathrm{H}+\mathrm{H}+\mathrm{M} \rightarrow \mathrm{H}_{2}+\mathrm{M}$ & $k=6.4 \times 10^{17} T^{-1^{\mathrm{a}}}$ & $k=3.62 \times 10^{18} T^{-1^{\mathrm{b}}}$ \\
$\mathrm{H}^{+}+\mathrm{e}^{-}+\mathrm{M} \rightarrow \mathrm{H}+\mathrm{M}$ & $k=5.26 \times 10^{26} T^{-5 / 2^{\mathrm{c}}}$ & $k=1.00 \times 10^{27} T^{-5 / 2^{\mathrm{c}}}$ \\
$\mathrm{H}_{2}+\mathrm{e}^{-} \rightarrow \mathrm{H}+\mathrm{H}+\mathrm{e}^{-}$ & $k=1.91 \times 10^{11} T e^{(-203 / \mathrm{RT})^{\mathrm{c}}}$ & $k=1.91 \times 10^{9} T^{(-203 / \mathrm{RT})^{\mathrm{c}}}$ \\
$\mathrm{H}^{-}+\mathrm{H} \rightarrow \mathrm{H}_{2}+\mathrm{e}^{-}$ & $k=7.83 \times 10^{14^{\mathrm{d}}}$ & $k=7.83 \times 10^{16^{\mathrm{d}}}$ \\
$\mathrm{H}^{+}+\mathrm{e}^{-} \rightarrow \mathrm{H}+\mathrm{h} \nu$ & $k=3.77 \times 10^{13} T^{-0.58^{\mathrm{e}}}$ & $k=3.77 \times 10^{15} T^{-0.58^{\mathrm{e}}}$ \\
$\mathrm{H}+\mathrm{e}^{-} \rightarrow \mathrm{H}+\mathrm{h} \nu$ & $k=7.5 \times 10^{4} T^{\mathrm{c}}$ & $k=7.5 \times 10^{4} T^{\mathrm{c}}$ \\
$\mathrm{H}^{+} 2 \mathrm{e}^{-} \rightarrow \mathrm{H}+\mathrm{e}^{-}$ & $k=7.08 \times 10^{39} T^{-9 / 2^{\mathrm{c}}}$ & $k=7.08 \times 10^{40} T^{-9 / 2^{\mathrm{c}}}$ \\
$\mathrm{H}^{+}+\mathrm{H}^{-} \rightarrow \mathrm{H}+\mathrm{H}$ & $k=1.30 \times 10^{18} T^{-1 / 2^{\mathrm{f}}}$ & $k=1.30 \times 10^{19} T^{-1 / 2^{\mathrm{f}}}$ \\
\hline \hline
\end{tabular}

${ }^{a}$ From Ref. 5. ${ }^{b}$ From Ref. 6. ${ }^{c}$ From Ref. 7, with calculation. ${ }^{d}$ From Ref. 8. ${ }^{\mathrm{e}}$ From Ref. 9, with calculations. ${ }^{\mathrm{f}}$ From Ref. 10 . 
literature and discussions with chemical kinetics researchers. Rate set 2 generally employed a faster set of rates or modified baseline rates. The rate set 2 modified baseline rates were obtained by increasing the rate set 1 baseline rates by either the experimental error or, if the experimental error was not quoted, by a factor of 10 . When more than one rate was found for a particular reaction, and it was not possible to discern the better, the faster rate was used in rate set 2 .

Since recombination was under study, the rates were all increased in the recombination direction. For the equation, $\mathrm{H}_{2}+\mathrm{e}^{-} \rightarrow \mathrm{H}+\mathrm{H}+\mathrm{e}^{-}$, no data were found in the recombination direction. For this reason, the equation and the kinetic rate are in the reverse direction. The $\mathrm{H}+\mathrm{e}^{-} \rightarrow \mathrm{H}^{-}+\mathrm{h} \nu$ reaction rate, which was judged insignificant, was accidentally left in both reaction sets. It should have been omitted.

\section{Analytical Procedure}

The performance (thrust/mass flow combination) of a thruster is dependent upon the expansion of the exhaust gases through a nozzle, which is in turn controlled by the rates of the chemical reactions. The ODK program uses this reaction data to predict rocket performance is terms of specific impulse. The one-dimensional equilibrium (ODE) nozzle analysis version of TDK supplies the chemical equilibrium gas composition and the pressure field needed for the kinetics calculations in ODK. The ODE/ODK code is a standard propulsion analytical tool and is used throughout government and industry. Details on its specifies are readily available in another publication. ${ }^{4}$

As written, the computer program provides thermodynamic data on the species $\mathrm{H}, \mathrm{H}^{+}$, and $\mathrm{H}^{-}$to a temperature of 5000 $\mathrm{K}$. This analysis required thermodynamic data for the species $\mathrm{H}_{2}, \mathrm{H}, \mathrm{H}^{+}, \mathrm{H}^{-}$, and $\mathrm{e}^{-}$to the maximum temperature under consideration $(18,000 \mathrm{~K})$. The additional thermodynamic property data were obtained directly from partition function calculations, similar to the method described by Patch. ${ }^{11}$

To use ODK for the nonequilibrium flow analysis, the forward reaction rates (Table 2) were converted to the reverse form $\left(k_{r}\right)$ through the equilibrium relationship: $K=k_{f} / k_{r}$, where $K$ is the equilibrium constant and $k_{f}$ is the forward rate. The reaction rate for each reaction was input through the Arrhenius parameters $A, N$, and $B$. For the three-body reactions within the set, third-body efficiency values were assumed to be unity unless explicitly indicated in Table 2 .

The analysis was conducted using a number of variable parameters other than the rates. Table 3 provides a list of the parameters and their range of values.

\section{Results and Discussion}

With the large number of independent parameters available for parametric variation, a multitude of relationships can be examined. The key dependent parameter of interest is the specific impulse and, therefore, the following discussion will concentrate on it and the various independent parameters that influence it. Primarily, the effects of chamber temperature, nozzle area ratio, and kinetic rate set will be examined.

The following discussion is aimed at providing information on performance possibilities for a laser thruster. A wealth of calculations, other than those shown, will be made available in a subsequent publication. ${ }^{12}$ The data do not reveal trends other than what one would expect, but they do provide a quantitative reference for assessments. A summary of the results includes:

Table 3 Analytical parameters

\begin{tabular}{lc}
\hline \multicolumn{1}{c}{ Parameters } & \multicolumn{1}{c}{ Value } \\
\hline Stagnation pressure, $P c, \mathrm{MPa}$ & $0.101,0.304,1.01$ \\
Stagnation temperature $T c, \mathrm{~K}$ & $3000,6000,9000,12,000$ \\
& $15,000,18,000$ \\
Area ratio $A e / A^{*}$ & $1,5,10,15,20,25$ \\
Nozzle half-angle $\theta, \mathrm{deg}$ & $7,14,21$ \\
\hline
\end{tabular}

1) For the nozzle types under consideration, achievable specific impulse is a strong function of the kinetic rates.

2) The nozzle area ratio effect on specific impulse is not large for the low chamber pressures anticipated. Thus, a largearea-ratio nozzle may not be useful for these thrusters. A short description of some of the significant parametric variations is included in the following discussion.

\section{Specific Impulse/Kinetic Rate}

Figure 3 is typical of all the predictions of specific impulse as a function of chamber temperature. The expected operating bulk temperature range for a laser thermal thruster is between 3000 and $6000 \mathrm{~K}$. As previously discussed, the range is related to the chamber heat loads amenable to current technology. At these values, the best available kinetics (rate set 1) indicate that the flow is very nearly frozen and that specific impulse values are several hundred seconds below the typically quoted equilibrium values. The explanation for the difference is the lack of recovery of molecular recombination energy in frozen flow. Figure 4 demonstrates this by showing the $\mathrm{H}$ atom concentration as a function of area ratio (axial location) for the 7 $\mathrm{deg}$, half-angle nozzle. For the full expansion (25:1), 20\% of the potential recombinant energy is lost. This performance decrease is only slightly recovered by the molecular weight advantage. Thus, a significant specific impulse difference is noted between equilibrium flow and the best kinetic flow prediction. The effect of the various rates changes with temperature; as we increase the chamber temperature from below to above $6000 \mathrm{~K}$, we see the ionization rates begin to

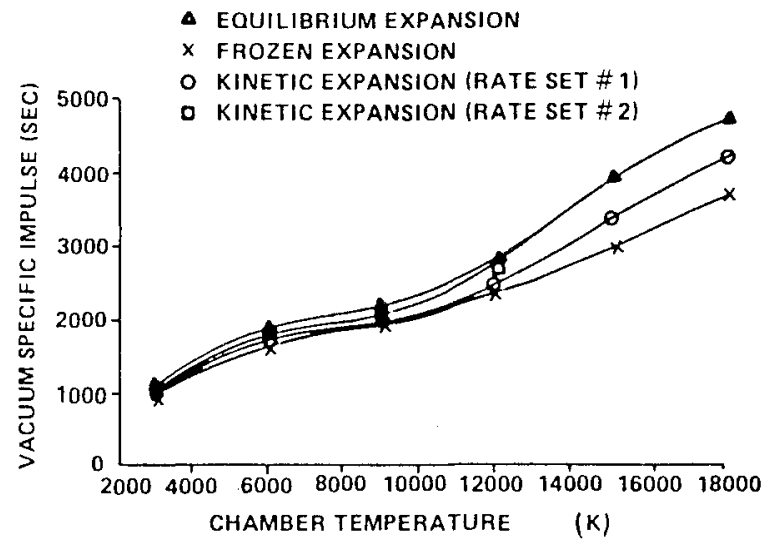

Fig. 3 Hydrogen thruster kinetic performance $(P c=0.101 \mathrm{MPa}$, nozzle half-angle $=7 \mathrm{deg}, A e / A^{*}=25$ ).

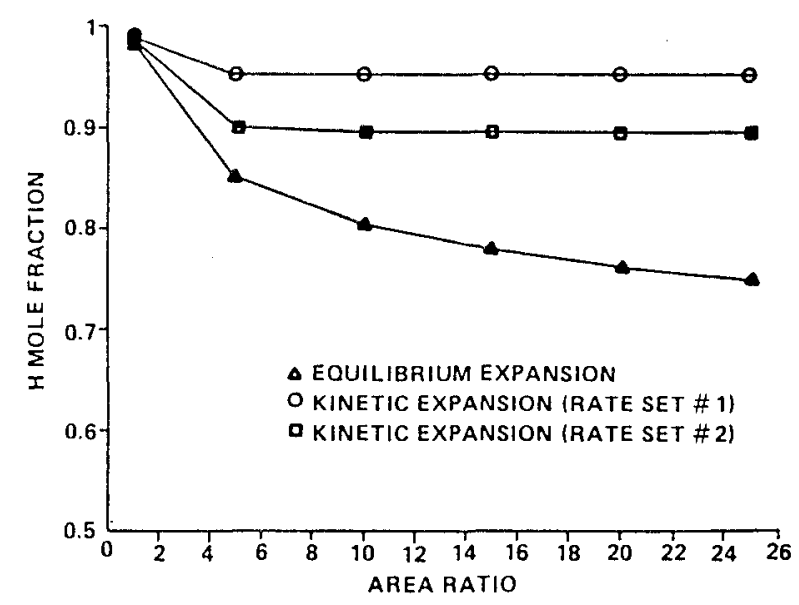

Fig. 4 Hydrogen thruster kinetic performance $(P c=0.101 \mathrm{MPa}$, $T c=6000 \mathrm{~K}$, nozzle half-angle $=7 \mathrm{deg}$ ). 


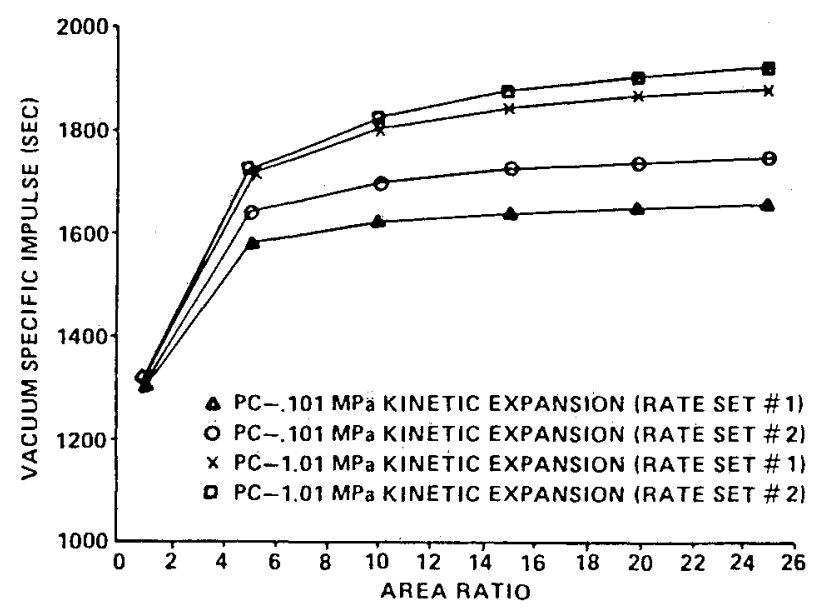

Fig. 5 Hydrogen thruster kinetic performance $(T c=6000 \mathrm{~K}$, nozzle half-angle $=14 \mathrm{deg}$ ).

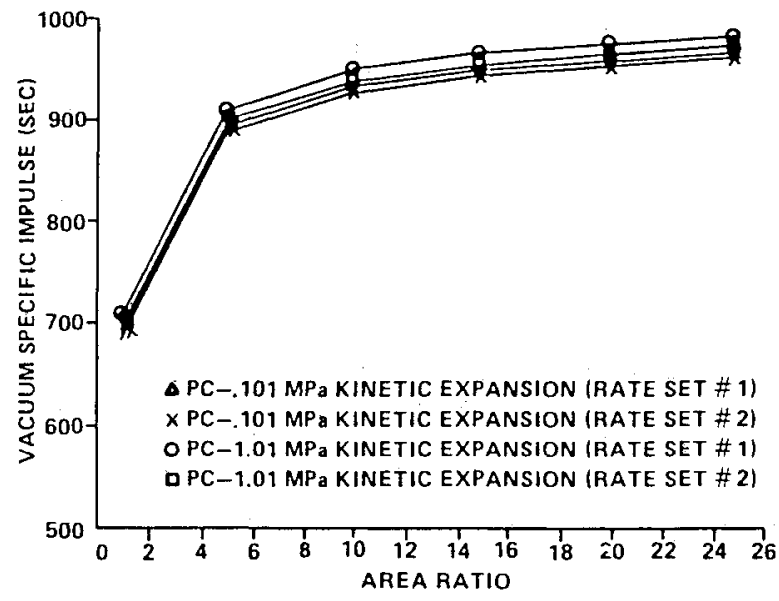

Fig. 6 Hydrogen thruster kinetic performance $(T c=3000 \mathrm{~K}$, nozzle half-angle $=14 \mathrm{deg}$ ).

weigh more heavily than the atomic recombination. This accounts for the characteristic flattening seen in Fig. 3.

\section{Specific Impulse/Chamber Pressure}

The plasma devices under development at Marshall Space Flight Center operate at low pressure, 0.05-1 MPa (0.5-10 atm). The effect of pressure on performance is determined by the chemical kinetic rates. This is clearly indicated in Fig. 5 where specific impulse increases 240 seconds as pressure increases from 0.1 to $1 \mathrm{MPa}$ for rate set $1($ area ratio $=25)$. For rate set 2 , the specific impulse increases only $170 \mathrm{~s}$. Due to the effect of the chemical kinetic rates, the specific impulse difference between rate sets 2 and 1 decreases from $100 \mathrm{~s}$ at a pressure of $0.1 \mathrm{MPa}$ to $30 \mathrm{~s}$ at a pressure of $1 \mathrm{MPa}$ (area ratio $=25$ ). Thus, the effect of increasing pressure is to make the rates more equivalent. This would be anticipated. At lower chamber temperatures, the effect becomes increasingly less significant, as can be discerned in Fig. 6 for a $3000 \mathrm{~K}$ chamber temperature.

\section{Specific Impulse/Area Ratio}

In general, a space nozzle is expected to be large in area ratio to permit maximum performance to be realized. The laser thermal thruster operates at low pressure and the impact of the flow freezing greatly reduces the utility of a high-arearatio nozzle. Figure 7 demonstrates that effect. The perfor-

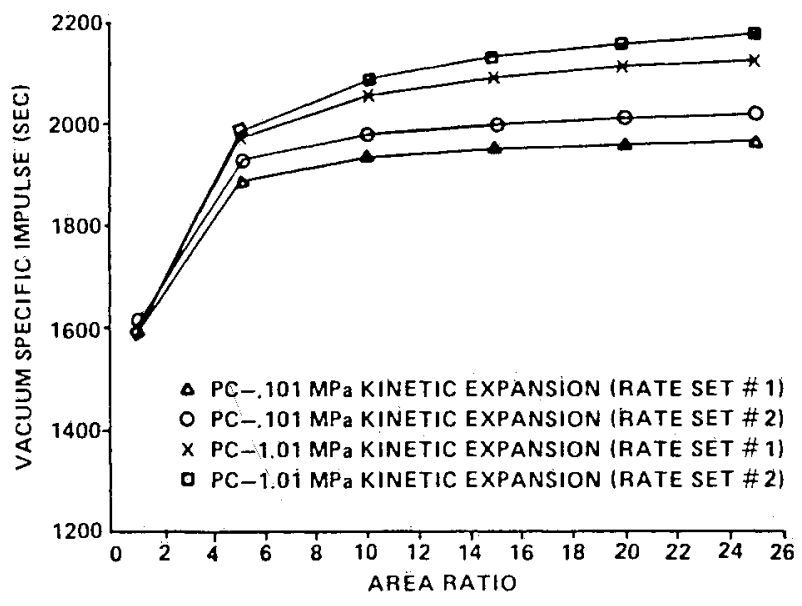

Fig. 7 Hydrogen thruster kinetic performance $(T c=9000 \mathrm{~K}$, nozzle half-angle $=14 \mathrm{deg}$ ).

mance is seen to be a strong function of area ratio of $A e / A^{*}=1-5$, but for larger area ratios has a minimal effect, at least in the context of large specific impulse. Thus, a laser thermal thruster would not require a high-expansion-ratio nozzle.

\section{Conclusions}

The previous section presented only a small percentage of the computations performed. The significance of the data is the expectation one can have for the performance of an actual laser thermal thruster. Current research and technology efforts at MSFC and elsewhere are pursuing the laser thermal thruster concept in search of the $2500 \mathrm{~s}$ specific impusle potential. This study is an attempt to examine the realism of that number for the purpose of evaluating such a system for orbital transfer applications. The presented specific impulse is an overestimate of the true system value since the laser resupply was not included in the mass flow parameter of the calculation. Nevertheless, the thruster-only portion of the system evaluation is in itself significant.

In that evaluation, one must account for kinetic effects, heat losses to the hardware, flow divergence. nonuniform pressure, and temperature profile and boundary-layer effects. This study examines only the first entity, the effect of chemical nonequilibrium. From the data presented in the previous section, it is obvious that the kinetic rates will signifcantly reduce the performance of a hydrogen laser thermal thruster. In fact, it appears that the performance level will be closer to that for frozen rather than equilibrium flow. When one also accounts for a practical bulk temperature limit $(5000 \mathrm{~K})$ for a wellstirred system, a specific impulse loss of several hundred seconds is to be expected. Based upon the predictions obtained with the ODK code, the practical upper limit for a hydrogen laser thermal thruster should be set at 1300-1900 s rather than 2500 s. Attempts to drive the bulk temperature and performance past that range of values does not appear worthwhile. Research in progress by the authors and several colleagues should address many of the other loss mechanisms and the overall laser/laser thruster system performance.

\section{Acknowledgments}

The authors acknowledge Mr. Ed Paff for performing the computations and Dr. S.C. Kurzius for providing valuable information on the various reactions and reaction rates.

\section{References}

${ }^{1}$ VanZandt, D.M., McCay, T.D., and Eskridge, R.H., "An Experimental Study of Laser Supported Hydrogen Plasmas," AIAA Paper 84-1572, June 1984. 
${ }^{2}$ McCay, T.D. and Thoenes, J., “Numerical Modeling of Laser Thermal Propulsion Flows," AIAA Progress in Astronautics and Aeronautics: Orbit-Raising and Maneuvering Propulsion-Research Status and Needs, Vol. 89, edited by L.H. Caveny, AIAA, New York, 1983. p. 149-166.

${ }^{3}$ Jones, L.W. and Keefer, D.R., "The NASA Laser Propulsion Project: An Update," AIAA Paper 81-1248, June 1981.

${ }^{4}$ Nickerson, G.R., Coats, D.E., and Bartz, J.L., "TDK-TwoDimensional Kinetics Research Computer Program,", Ultrasystems, Irvine, California, Dec. 1973.

${ }^{5}$ Baulch, D.L., Drysdale, D.D., Horne, D.G., and Lloyd, A.C., Evaluated Kinetic Data for High Temperature Reactions, Vol. 1, Butterworths, London, 1972.

${ }^{6}$ Jensen, D.E. and Kurzius, S.C., "Rate Constants for Calculations on Nozzle and Rocket Exhaust Flow Fields," AeroChem Research Laboratories, Inc., Princeton, NJ, Rept. TP-149, March 1967.

${ }^{7}$ Tal Rose, V.L. and Karachevtsev, G.V., "Elementary Reactions in Low Temperature Plasma," Reactions Under Plasma Conditions,
Vol. II, edited by M. Venugopalan, Wiley, New York, 1971. pp. 35-140.

${ }^{8}$ Schmeltekopf, A.L., Fehsenfeld, F.C, and Ferguson, E.E., "Laboratory Measurement of the Rate Constant for $\mathrm{H}^{-}+\mathrm{H} \rightarrow \mathrm{H}_{2}+\mathrm{e}^{-}$ Astrophysical Journal, Vol. 148, 1967, p. L155.

${ }^{9}$ Bates, D.R., Kingston, A.E. and McWhirter, R.W.P., "Recombination Between Electrons and Atomic Ions II. Optically Thick Plasmas" Proceeding of the Royal Society, London, Vol. A270, 1962, p. 155.

${ }^{10}$ Bates, D.R. and Boyd, T.J.M., "Inelastic Heavy Particle Collisions involving the Crossing of Potential Energy Curves IV. Ionic Recombination" Proceedings of the Physical Society, London, Vol. A69, 1956, p. 910.

${ }^{11}$ Patch, R.W., "Thermodynamic Properties and Theoretical Rocket Performance of Hydrogen to $100000 \mathrm{~K}$ and $1.01325 \times 10^{8}$ $\mathrm{N} / \mathrm{m}^{2}, "$ NASA SP-3069, 1971.

${ }^{12}$ Dexter, C.E. and McCay, T.D., "Performance Loss for a Hydrogen Fueled Laser Thermal Rocket," NASA TM, to be published.

\title{
From the AIAA Progress in Astronautics and Aeronautics Series ...
}

\author{
SATELLITE COMUNICATIONS: \\ FUTURE SYSTEMS-v. 54 \\ ADVANCED TECHNOLOGIES-v. 55
}

\author{
Edited by David Jarett, TRW, Inc.
}

Volume 54 and its companion Volume 55, provide a comprehensive treatment of the sateilite communication systems that are expected to be operational in the 1980's and of the technologies that will make these new systems possible. Cost effectiveness is emphasized in each volume, along with the technical content.

Volume 54 on future systems contains authoritative papers on future communication satellite systems in each of the following four classes: North American Domestic Systems, Intelsat Systems, National and Regional Systems, and Defense Systems. A significant part of the material has never been published before. Volume 54 also contains a comprehensive chapter on launch vehicles and facilities, from present-day expendable launch vehicles through the still developing Space Shuttle and the Intermediate Upper Stage, and on to alternative space transportation systems for geostationary payloads. All of these present options and choices for the communications satellite engineer. The last chapter in Volume 54 contains a number of papers dealing with advanced system concepts, again treating topics either not previously published or extensions of previously published works.

Volume 55 on advanced technologies presents a series of new and relevant papers on advanced spacecraft engineering mechanics, representing advances in the state of the art. It includes new and improved spacecraft attitude control subsystems, spacecraft electrical power, propulsion subsystems, spacecraft antennas, spacecraft RF subsystems, and new earth station technologies. Other topics are the relatively unappreciated effects of high-frequency wind gusts on earth station antenna tracking performance, multiple-beam antennas for higher frequency bands, and automatic compensation of crosspolarization cuoupling in satellite communication systems.

With the exception of the first "visionary" paper in Volume 54, all of these papers were selected from the 1976 AIAA/CASI 6th Communication Satellite Systems Conference held in Montreal, Cnada, in April 1976, and were revised and updated to fit the theme of communication satellites for the 1980's. These archive volumes should form a valuable addition to a communication engineer's active library.

Volume 54, Published in 1977, 541 pp., $6 \times 9$ illus., $\$ 25.00 \mathrm{Mem} ., \$ 45.00$ List

Volume 55, Published in 1977, 489 pp., $6 \times 9$ illus., $\$ 25.00$ Mem., $\$ 45.00$ List

TO ORDER WRITE: Publications Order Dept., AIAA, 1633 Broadway, New York, N.Y. 10019 